\title{
Article \\ Prediction of Preterm Delivery from Unbalanced EHG Database
}

\author{
Somayeh Mohammadi Far ${ }^{1, *(\mathbb{D}}$, Matin Beiramvand ${ }^{2}$, Mohammad Shahbakhti ${ }^{3}{ }^{\mathbb{C}}$ and Piotr Augustyniak ${ }^{1} \mathbb{C}$ \\ 1 AGH University of Science and Technology, 30059 Krakow, Poland; august@agh.edu.pl \\ 2 Department of Biomedical Engineering, Dezful Branch, Islamic Azad University, Dezful 313, Iran; \\ matin.beiramvand@gmail.com \\ 3 Biomedical Engineering Institute, Kaunas University of Technology, 51423 Kaunas, Lithuania; \\ mohammad.shahbakhti@ktu.edu \\ * Correspondence: far@agh.edu.pl
}

check for updates

Citation: Mohammadi Far, S.; Beiramvand, M.; Shahbakhti, M.; Augustyniak, P. Prediction of Preterm Delivery from Unbalanced EHG Database. Sensors 2022, 22, 1507. https://doi.org/10.3390/s22041507 Academic Editor: Juan Pablo Martínez

Received: 15 December 2021 Accepted: 11 February 2022 Published: 15 February 2022

Publisher's Note: MDPI stays neutral with regard to jurisdictional claims in published maps and institutional affiliations.

Copyright: () 2022 by the authors Licensee MDPI, Basel, Switzerland. This article is an open access article distributed under the terms and conditions of the Creative Commons Attribution (CC BY) license (https:// creativecommons.org/licenses/by/ $4.0 /)$.

\begin{abstract}
Objective: The early prediction of preterm labor can significantly minimize premature delivery complications for both the mother and infant. The aim of this research is to propose an automatic algorithm for the prediction of preterm labor using a single electrohysterogram (EHG) signal. Method: The proposed method firstly employs empirical mode decomposition (EMD) to split the EHG signal into two intrinsic mode functions (IMFs), then extracts sample entropy (SampEn), the root mean square (RMS), and the mean Teager-Kaiser energy (MTKE) from each IMF to form the feature vector. Finally, the extracted features are fed to a k-nearest neighbors $(\mathrm{kNN})$, support vector machine (SVM), and decision tree (DT) classifiers to predict whether the recorded EHG signal refers to the preterm case. Main results: The studied database consists of 262 term and 38 preterm delivery pregnancies, each with three EHG channels, recorded for $30 \mathrm{~min}$. The SVM with a polynomial kernel achieved the best result, with an average sensitivity of $99.5 \%$, a specificity of $99.7 \%$, and an accuracy of $99.7 \%$. This was followed by DT, with a mean sensitivity of $100 \%$, a specificity of $98.4 \%$, and an accuracy of $98.7 \%$. Significance: The main superiority of the proposed method over the state-of-the-art algorithms that studied the same database is the use of only a single EHG channel without using either synthetic data generation or feature ranking algorithms.
\end{abstract}

Keywords: preterm labor; prediction; electrohysterogram; empirical mode decomposition; support vector machine

\section{Introduction}

Preterm birth, also referred to as premature delivery, is defined as giving birth sooner than after 37 weeks of gestation. Preterm delivery is considered a complex condition that occurs due to several biological, mental, and clinical factors, such as, but not limited to, ascending infection, hypoxic-ischemic damage to the uteroplacental unit, chronic stress and fetal and/or uterine developmental malformations, maternal stress, depression, multiple gestations, abortion and short cervical lengths, surgery, ethnicity, and lifestyle [1,2]. However, the extent to which these factors are associated with premature delivery has not yet been proven, as almost $50 \%$ of preterm births happen without observation of the mentioned factors [3].

According to the World Health Organization (WHO), preterm birth is the leading cause of fetal morbidity and mortality, and it is increasing all around the world. A recent report from the WHO shows that more than 15 million neonates are delivered prematurely, of which 1 million die each year due to complications [4]. Even the survivors are exposed to various lifelong disabilities, such as, but not limited to, learning difficulties and vision or hearing impairments. Regardless of its complications, the price of medical care for preterm babies imposes a significant financial burden on the family and society, as it costs 5- to 10-times more than a term birth [5]. Thus, early prediction of preterm delivery, combined with appropriate medication to prevent this phenomenon, can greatly minimize 
the corresponding complications for both the mother and the baby, and reduce the economic load on public health systems.

To predict preterm labor, several physiological measurements, such as tocodynamometer [6], ultrasound [7], fetal fibronectin [8], and internal uterine pressure [9] have been studied. Despite adequate performance, the mentioned measurements are either subjective or invasive [10]. Recently, the analysis of electrical representation of uterus contractions collected from the abdominal surface of pregnant women, known as electrohysterography (EHG), has been evidenced as a reliable tool for the prediction of premature delivery, as it is a non-invasive procedure that can be automated to reduce human intervention [11-13].

To profile EHG signals for preterm labor prediction, a wide range of temporal and spectral measurements have been investigated [14-16]. Khalil and Duchene [17] presented a hybrid algorithm based on the dynamic cumulative sum and multiscale decomposition to detect different events of EHG signals. Moslem et al. [18] investigated the irregularity of EHG signals by sample entropy (SampEn) and showed its suitability for the discrimination of term and preterm pregnancies. Diab et al. [19] examined time reversibility, SampEn, Lyapunov exponents, and delay vector analysis to classify term and preterm EHG signals, and reported that time reversibility gives the best results. Fele-Zorz et al. [20] compared linear and nonlinear features to analyze EHG recordings for the identification of term and preterm deliveries, demonstrating that non-linear ones are more appropriate. Using wavelet package decomposition (WPD), Alamedine et al. [21] examined the adaptability of several spectral features for term-preterm labor classification, and concluded that the mean power frequency gives the best results. Ahmed et al. [22] showed the superiority of multivariate multiscale fuzzy entropy over multivariate multiscale entropy for the classification of term and preterm cases using EHG signals. Horoba et al. [23] studied various temporal and spectral features of EHG signals, and reported the median frequency as the most suitable feature for distinguishing term and preterm records. Yet, the main limitation of the above-mentioned studies is that they consider only one measure for the discrimination of term and preterm cases, compromising the performance of the learning method [24].

\section{Related Works}

One of the most-studied EHG-related delivery databases is Term-Preterm ElectroHysteroGram DataBase (TPEHG DB), which contains 262 term and 38 preterm labor records, each with three channels [20]. A detailed description of the database is provided in Section 4.1. One of the greatest challenges for the aforementioned database is the imbalanced class distribution of term and preterm EHG cases, which was resolved by employing data-generation methods such as the synthetic minority oversampling technique (SMOTE) [25] or the adaptive synthetic sampling approach (ADASYN) [26]. Fergus et al. [27] extracted the peak frequency, median frequency, root mean square (RMS), and SampEn from a single EHG channel band-pass filtered at $0.34-1 \mathrm{~Hz}$, and employed SMOTE to overcome the imbalance problem. The best result was achieved by the polynomial classifier with an average sensitivity of $97 \%$, a specificity of $90 \%$, and an area under the curve (AUC) of 0.95. With features similar to [27] calculated from a single EHG channel band-pass filtered at $0.3-3 \mathrm{~Hz}$ plus the min-max oversampling method, Hussain et al. [28] reported $91.2 \%$ for sensitivity, $94.5 \%$ for specificity, and $92.7 \%$ for accuracy with the selforganized network inspired by the immune algorithm (SONIA) classifier. Smrdel et al. [29] used the adaptive autoregressive method to estimate the median frequency and SampEn from a single EHG channel band-pass filtered at 0.3-4 Hz. After employing SMOTE, quadratic discriminant analysis and a support vector machine showed accuracies of $86 \%$ and $87 \%$, respectively. In another study, Fergus et al. [30] extracted SampEn, waveform length, log detector, and variance from a single EHG channel similar to [27]. Using SMOTE for the data balancing, the authors reported an AUC of 0.94 , a sensitivity of $91 \%$, and a specificity of $84 \%$, achieved by mixture of a Levenberg-Marquardt-trained feed-forward neural network, a radial basis function neural network, and random neural network classi- 
fiers. Peng et al. [31] investigated the appropriateness of 31 temporal and spectral features extracted from three EHG channels, which were collected before the 26th week of gestation. After selecting 15 features and employing ADASYN, a random forest classifier showed an accuracy of $93 \%$, a sensitivity of $89 \%$, and a specificity of $97 \%$. Degbedzui et al. [32] employed autoregressive modeling to extract a new measure, called centroid frequency estimation, from a single EHG channel. After employing ADASYN, an accuracy of 99.72\%, a specificity of $99.96 \%$, and a sensitivity of $99.48 \%$ was achieved by the SVM classifier. Ye-Lin et al. [33] extracted 203 temporal, spectral, and nonlinear features from 326 multichannel EHG recordings and employed SMOTE to balance the database. After feature selection by a genetic algorithm, the authors reported a mean F1 score of $92.04 \%$ using an ensemble classifier. Later on, Ye-Lin et al. [5] showed the efficiency of entropy measures for the classification of the term-preterm EHG recordings by a linear discriminant analysis (LDA) classifier with an average F1 score of 90.1\%. Yang et al. [34] extracted RMS, median frequency, peak frequency, and sample entropy from the EHG signals, and applied SMOTE for overcoming the data imbalance issue. After employing five classifiers, the authors reported the best accuracy, of $85 \%$, by the gradient boosting classifier (GBC). Xu et al. [35] presented a network theory-based algorithm for the prediction of preterm labor. Using a partition-synthesis method for overcoming the data balancing and SVM for classification, an accuracy of $91 \%$ was achieved.

As EHG signals are generated from a huge amount of intricately interconnected cells, uterus contractions are expected to display strong non-stationarity [20]. Consequently, employing non-stationary algorithms for feature extraction was expected to improve the classification results. The most prominent example of such methods is empirical mode decomposition (EMD) [36], which splits the input signal into several frequency components (ordered from high to low), called intrinsic mode functions (IMFs), without requiring any prior knowledge such as basis functions in the wavelet transform. For this reason, some researchers performed EMD on EHG data, then extracted features from the decomposed components. Ren et al. [37] used the Shannon entropy of the first ten decomposed IMFs to form the feature vector. After applying SMOTE, several classifiers were used to discriminate term and preterm deliveries. According to the authors, the best result was achieved based on the Adaboost classifier with an AUC of 0.986. N. Sadi et al. [38] used a balanced data set of EHG records (26 term and 26 preterm records) and extracted seven features from the IMF3 and IMF6 of two EHG channels. After employing a linear SVM classifier, an average accuracy of $95.70 \%$, a sensitivity of $98.40 \%$, a specificity of $93 \%$, and an AUC of 0.95 were achieved. Acharya et al. [39] employed EMD to extract 11 IMFs from EHG records that were subsequently decomposed by WPD to 6 levels. After ranking significant features and employing ADASYN, SVM achieved an accuracy of $96.25 \%$, a sensitivity of $95.08 \%$ and a specificity of $97.33 \%$. Khan et al. [40] extracted nine features from the second to fifth IMFs. After using ADASYN for data balancing and SVM for classification, an accuracy of $98 \%$ was obtained.

While the mentioned investigations showed satisfactory results for the prediction of preterm labor, the majority of them employed synthetic data generation to overcome the imbalance problem, which may cause misleading results $[11,29,30]$, i.e., if an algorithm detects preterm labor cases with a sensitivity of $90 \%$ after data balancing, it is not clear how much of that $10 \%$ of misclassified preterm cases are related to real data. Indeed, if such a misclassification is strongly related to the real data, not the synthetically genereated ones, the performance may not be reliable. To this aim, this research proposes an automatic algorithm to predict preterm labor from a single EHG channel without employing any synthetic data-generation algorithms. Our hypothesis is that EHG records related to preterm labor contain stronger contractions than term ones; therefore, instead of extracting many features, we consider only three features that properly address such differences. In addition, given our hypothesis-that EHG signals related to preterm labor cases have stronger contractions - feature extraction is better to be performed on EHG signals decomposed by EMD, where the EHG signal is represented by several components with different frequency 
ranges. After the feature extraction, k-nearest neighbors (kNN), SVM, and decision tree (DT) classifiers are employed to investigate the effectiveness of the proposed features.

\section{Methods}

\subsection{The Proposed Method}

The block diagram of the proposed method is shown in Figure 1. In short, the following procedures will be employed: (i) two IMFs are extracted from EHG signals; (ii) three features are extracted from these IMFs to form the feature vector; (iii) the extracted features are fed to three classifiers for studying the best possible discrimination of the term and preterm cases. The following subsections explain the proposed method in detail.

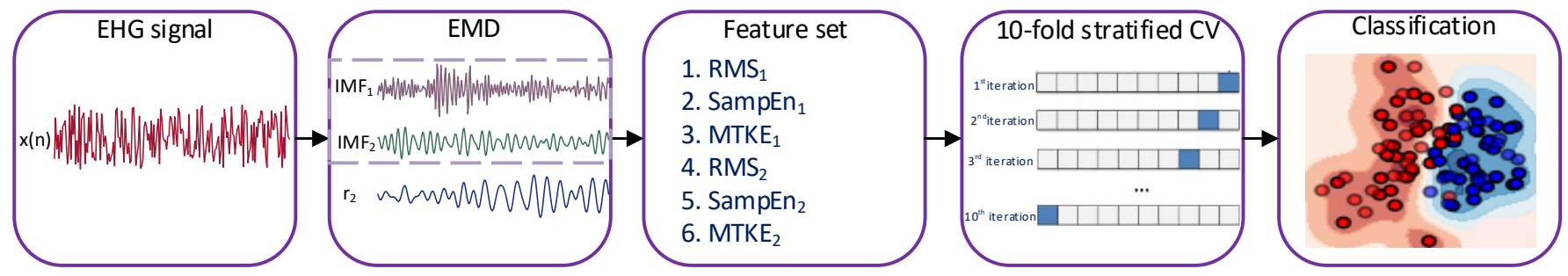

Figure 1. The block diagram for the discrimination of the deliveries.

\subsection{Empirical Mode Decomposition}

The basis of EMD is to decompose the input signal $x(n)$ into $m$ number of intrinsic mode functions (IMFs) and one residual signal $r(n)$, where the original signal can be reconstructed as follows:

$$
x(n)=\sum_{j=1}^{m} I M F_{j}+r_{m}(n) .
$$

Indeed, EMD decomposes the input signal from high to low frequency components as the level of decomposition, $j$, increases. Since the input signal is decomposed in the time domain, and the length of the decomposed components and the original signal is equal, EMD preserves the characteristics of varying frequencies [36]. An IMF is defined as a function that satisfies the two conditions:

1. In the whole data set, the number of extrema and zero-crossings must either be equal or differ at most by one;

2. At any point, the mean value of the envelope defined by the local maxima and the envelope defined by the local minima is zero.

The second condition is ideal and may be not achieved in practice; therefore, it is controlled by a threshold. If the mean value of the lower and upper envelopes is below 0.2 , it is considered an IMF. The process of IMF extraction from $x(n)$, known as the sifting process, is described as follows:

1. Extract the local minima and local maxima from $x(n)$;

2. Create the upper and lower signal's envelopes using cubic spline;

3. Compute the local mean signal, $m_{1}(n)$, by averaging the upper and lower signal's envelopes;

4. Subtract $m_{1}(n)$ from $x(n)$ to obtain the first possible IMF candidate $y_{1}(n)=x(n)-$ $m_{1}(n)$.

Now, it should be investigated whether $y_{1}(n)$ fulfills IMF's conditions. If not, $y_{1}(n)$ is considered as a new signal, and steps $1-4$ have to be repeated. This process is continued $k$ times until $y_{1 k}(n)$ is chosen as the first IMF. In order to reach a series of IMFs, the residue, $r_{1}(n)$, should be generated as follows:

$$
r_{1}(n)=x(n)-I M F_{1} .
$$

Then, the sifting process is performed on $r_{1}(n)$ to obtain the second IMF. 
Considering the fact that EMD decomposes a signal from high- to low-frequency components, and that our hypothesis that EHG signals related to preterm labor cases might represent stronger contractions, i.e., show higher frequency components, we employ only the first two IMFs. In order to decompose EHG signals by EMD, each signal was windowed into 1-min segments, and the first two IMFs were extracted.

\subsection{Feature Extraction}

The first step for the true segmentation of term and preterm deliveries is to select distinctive features. Our presumption is that preterm EHG signals contain stronger contractions than term ones; thus, features able to represent this property should be extracted. In this paper, we extracted three features, RMS, SampEn [41], and mean Teager-Kaiser energy (MTKE) [42], from the first two IMFs. Although the adequacy of the employed features has been proven when directly extracted from EHG signals, better performance might be achieved if they are extracted from decomposed EHG signals, where high-frequency components, i.e., the first two IMFs, are the only ones considered. The motivation behind using these three features is their capability to distinguish stronger contractions, as EHG signals related to preterm cases are expected on the physiological background to show such behavior. Indeed, stronger contractions show higher amplitude (RMS), uncertainty (entropy), and energy (MTKE).

RMS is defined as the square root of the arithmetic mean of the squares of the values, expressed as follows:

$$
\mathrm{RMS}=\sqrt{\frac{1}{N} \sum_{n=1}^{N} x^{2}(n) .}
$$

As our assumption is that preterm EHG records contain stronger contractions, it is expected that the RMS of term and preterm records show a meaningful difference [14].

SampEn is a modification of approximation entropy without independence from the data length. For a given signal with a length of $n$, it can be expressed as the negative logarithm of conditional probability that two sequences are similar for $m$ point within a tolerance value $r$, excluding any self-matches. Thus, it can be represented as:

$$
\operatorname{SampEn}(m, r, n)=-\ln \left(\frac{A}{B}\right)
$$

where $A=\frac{(n-m-1)(n-m)}{2} A^{m}(r)$ and $B=\frac{(n-m-1)(n-m)}{2} B^{m}(r) . A^{m}(r)$ and $B^{m}(r)$ stand for the probabilities of two sequence matches for $m+1$ and $m$ points, respectively. SampEn represents the irregularity of the signal. As stronger contractions can also increase irregularity, it can be expected as a proper feature for the term and preterm segmentation $[5,18]$. SampEn requires two parameters to be adjusted before implementation, embedding dimension $m$ and scaling factor $r$. In this paper, we used $m=3$ and $r=0.15$, as suggested in [5].

TKE is a well-known tool for the detection of muscle-contraction onsets from electromyogram signals. In this paper, we use the mean of TKE as follows:

$$
\text { MTKE }=\mu\left(x^{2}(n)-x(n-1) x(n+1)\right),
$$

where $\mu$ represents the mean. The MTKE calculates the energy of a signal based on its amplitude and frequency content; hence, its higher average value can represent preterm cases [39].

As was already mentioned, each EHG signal is decomposed into two IMFs; therefore, 6 features from each channel are extracted. It should be noted that, after segmenting EHG signals into 1-min windows and the IMF extraction, each feature was computed from all those windows, and the average of them was considered as the final feature. In order to normalize the features' scale, each feature column is subtracted from its mean and divided by its standard deviation. 


\subsection{Classifiers}

Three classifiers were learned and tested to best distinguish the term and preterm delivery signs based on the above-presented features.

\subsection{1. k-Nearest Neighbors}

$\mathrm{kNN}$ is a simple supervised machine-learning algorithm, widely employed for classification and regression problems. The basis of $\mathrm{kNN}$ is to separate data points by using a distance function. Indeed, $\mathrm{kNN}$ performs the classification by the majority vote of neighbors, where each data point is attributed with a label that has the closest neighbors. Therefore, kNN presumes that resembling data are in close proximity. There are two parameters that influence the classification results of $\mathrm{kNN}$ : the number of neighbors $K$ and the distance metric. The determination of both parameters is an experimental task [43].

\subsubsection{Support Vector Machine}

SVM is one the most efficient supervised machine-learning algorithms that have been extensively used in dual classification problems. The main advantage of SVM is its capability to separate and handle multiple continuous and categorical variables. In general, SVM generates a hyperplane in multi-dimensional space to distinguish different classes. Compared to other classifiers, SVM's kernel-selection property provides a better solution to deal with complex data. Yet, the optimization of kernel parameters is a time-consuming procedure [44].

\subsubsection{Decision Tree}

The decision tree deals with the classification problem as a form of tree structures. It decomposes the database into smaller subsets that are incrementally developed. As the final results, decision and leaf nodes will be given, where decision nodes have two or more branches and leaf nodes represent the classification results [45]. Compared to the SVM, the classification result of DT depends on more required parameters in order to be tuned accurately. As a consequence, DT can be more operator-dependent.

\section{Evaluation}

\subsection{Data}

The EHG records included in the TPEHG DB database were collected from 1997 to 2005 at the University Medical Centre Ljubljana, Department of Obstetrics and Gynecology [20]. The TPEHG DB contains 300 EHG records, of which 262 records were of term and 38 records were of preterm deliveries. According to pregnancy weeks, these records are categorized into two groups, where 143 term and 19 preterm records were collected before the 26th week of gestation, and 119 term and 19 preterm records were collected during or after the 26th week of the gestation. Each record is comprised of three channels, recorded from four electrodes placed on the abdominal surface of pregnant women, as shown in Figure 2.

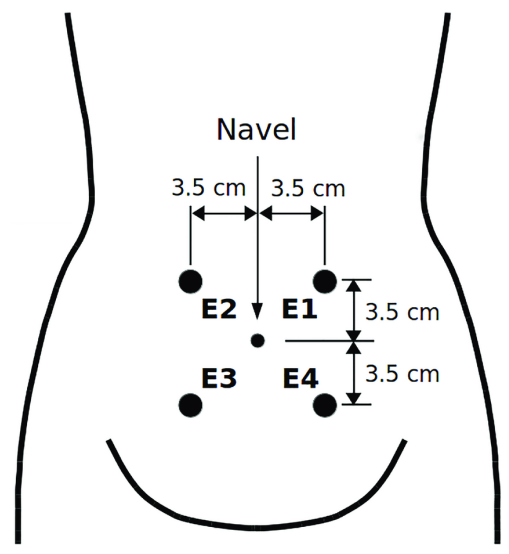

Figure 2. The placement of EHG electrodes, adopted from [46] 
Using differences in the electrical potentials of the electrodes, three channels were produced as $\mathrm{CH}_{1}=E 2-E 1, \mathrm{CH}_{2}=E 2-E 3$, and $\mathrm{CH}_{3}=E 4-E 3$. Each record lasted for $30 \mathrm{~min}$ and was sampled at $20 \mathrm{~Hz}$ with 16-bit resolution over a range of \pm 2.5 millivolts. After data collection, a fourth-order Butterworth band pass filter with cut-off frequencies of 0.08 and $4 \mathrm{~Hz}$ was employed to filter the raw EHG signals. To mitigate the transient effect of filtering, the first and last $180 \mathrm{~s}$ of each record were removed [20]. Figure 3 shows $1 \mathrm{~min}$ of the EHG signals from all three channels after filtering.

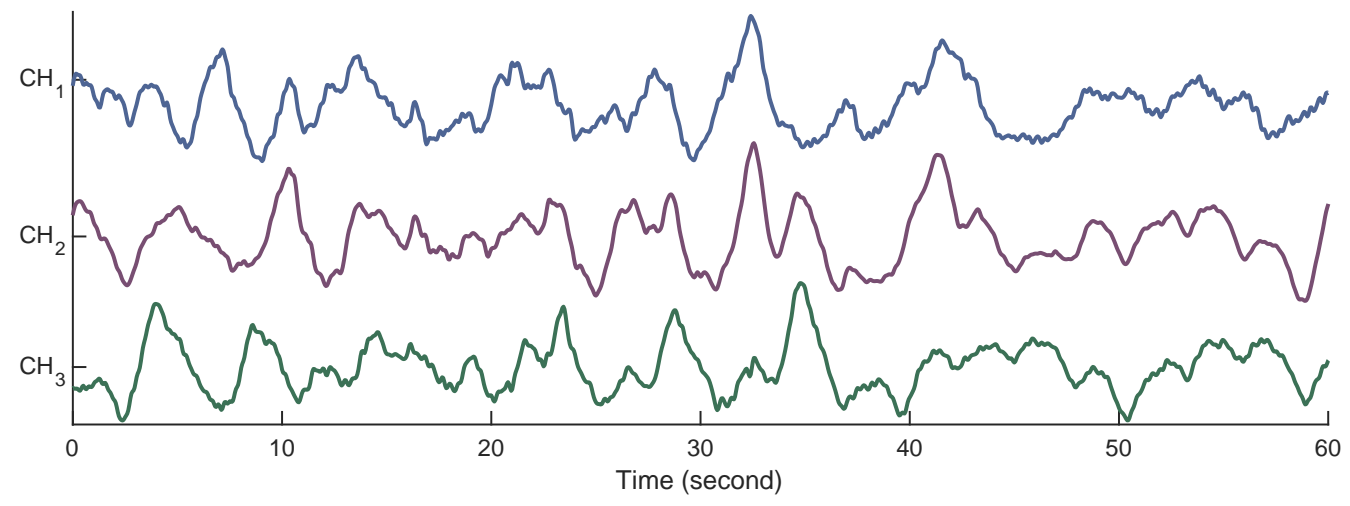

Figure 3. Examples of the EHG signals from all three channels.

\subsection{Imbalanced Database Issue}

The major problem of the TPEHG DB database is the imbalance of EHG data for term and preterm classes, as only $13 \%$ of data corresponds to preterm cases. In this situation, the classifiers may be biased against the preterm labor cases [47]. Indeed, by using the simple k-fold cross-validation, it is possible that few folds do not have preterm cases. The most straightforward strategy addressed in the literature is to generate synthetic preterm features by algorithms such as SMOTE or ADASYN. Yet, some studies argued that such a strategy may lead to misleading results $[11,29,30]$. To overcome this issue, we employed stratified k-fold cross-validation, which randomly splits the database into $k$ subsets and guarantees the existence of both classes in all subsets.

\subsection{Evaluation Metrics}

In order to evaluate the performance of the classifiers, the sensitivity $(\mathrm{Se})$, specificity $(S p)$, and accuracy $(A c c)$ are computed as follows:

$$
\begin{gathered}
S e=\frac{T P}{T P+F N} \times 100, \\
S p=\frac{T N}{T N+F P} \times 100, \\
A c c=\frac{T P+T N}{T P+T N+F P+F N} \times 100,
\end{gathered}
$$

where TP and FN represent the number of correctly and wrongly classified preterm cases, and $T N$ and FP stand for the number of correctly and wrongly classified term cases. To validate the performance of the classifiers, a 10-fold stratified cross-validation with 30 repetitions was performed. After splitting the data into 10 subsets, the training and testing procedures are performed in such a way that, each time, 9 subsets are used for training and 1 subset is used for testing. Consequently, the classification results are taken as the average of 10 repetitions for training and testing. It should be noted that during these 10 repetitions, the obtained testing results are independent of the previously trained classifier. After finding the best results for each classifier, the AUC is computed as follows:

$$
\mathrm{AUC}=\int S e(T)(1-S p)^{\prime}(T) d T,
$$


where $T$ is the threshold related to the binary classifier.

\section{Results and Discussion}

As mentioned in the state-of-art review, each classifier requires the accurate setting of parameters prior to performing the experiment. In this paper, we report the performance of each classifier with an alternation of the most-prominent parameter. The remaining parameters are set as the default. Compared to the state-of-art methods, we show the employed features better discriminate term and preterm cases, supporting our claim with quantitative results. Furthermore, the main superiority of the proposed method over the state-of-the-art algorithms is to employ only real EHG signals. The experiments were implemented in a MATLAB 2019 environment using a personal computer with a $3.2 \mathrm{GHz}$ core i7 CPU and 8-GB memory.

Figure 4 shows the distribution of the extracted features. As for $\mathrm{kNN}$, the distance metric and the number of neighbors should be specified first. According to the literature, the most common distance metric for kNN classifiers is Euclidean; thus, the number of neighbors plays the most important role. For this aim, a different number of neighbors, i.e., $2,4,8,10$, and 12, are examined. As shown in Table 1 , the best results were achieved from $\mathrm{CH}_{2}$ by 4 neighbors with a mean $\mathrm{Se}$ of $86.9 \%$, $\mathrm{Sp}$ of $98.0 \%$, and $A c c$ of $96.6 \%$, followed by 4 neighbors with a mean $S e$ of $86.1 \%, S p$ of $97.8 \%$, and $A c c$ of $96.3 \%$ from $C_{1}$.

Table 1. kNN performance for different channel configurations. The best obtained results are in bold.

\begin{tabular}{|c|c|c|c|c|}
\hline No. of K & Channel & Se & Sp & Acc \\
\hline \multirow{3}{*}{2} & CH1 & $81.2 \%$ & $95.1 \%$ & $93.9 \%$ \\
\hline & $\mathrm{CH} 2$ & $78.9 \%$ & $94.0 \%$ & $91.8 \%$ \\
\hline & $\mathrm{CH} 3$ & $77.3 \%$ & $95.4 \%$ & $93.2 \%$ \\
\hline \multirow{3}{*}{4} & CH1 & 86.1 \% & $97.8 \%$ & $96.3 \%$ \\
\hline & $\mathrm{CH} 2$ & $86.9 \%$ & $98.0 \%$ & $96.6 \%$ \\
\hline & $\mathrm{CH} 3$ & 82.9 \% & $97.6 \%$ & $96.7 \%$ \\
\hline \multirow{3}{*}{8} & $\mathrm{CH} 1$ & $82.1 \%$ & $98.7 \%$ & $96.6 \%$ \\
\hline & $\mathrm{CH} 2$ & $80.0 \%$ & $98.0 \%$ & $95.8 \%$ \\
\hline & $\mathrm{CH} 3$ & $81.3 \%$ & $99.6 \%$ & $97.2 \%$ \\
\hline \multirow{3}{*}{10} & $\mathrm{CH} 1$ & $79.2 \%$ & $98.8 \%$ & $96.2 \%$ \\
\hline & $\mathrm{CH} 2$ & $79.0 \%$ & $98.3 \%$ & $95.9 \%$ \\
\hline & $\mathrm{CH} 3$ & $80.0 \%$ & $99.6 \%$ & $97.1 \%$ \\
\hline \multirow{3}{*}{12} & $\mathrm{CH} 1$ & $77.1 \%$ & $98.9 \%$ & $96.0 \%$ \\
\hline & $\mathrm{CH} 2$ & $76.7 \%$ & $98.2 \%$ & $95.4 \%$ \\
\hline & $\mathrm{CH} 3$ & $78.0 \%$ & $99.6 \%$ & $96.8 \%$ \\
\hline
\end{tabular}

As for SVM, linear, radial basis function (RBF), and polynomial (Poly) kernels were used. It should be noted that kernel parameters that maximize the margin between term and preterm cases and minimize the misclassification rate were adjusted automatically in a MATLAB 2019 environment. Table 2 shows the classification results by SVM. As displayed, the best results were reached through $\mathrm{CH}_{1}$ features using a poly kernel with an average $\mathrm{Se}$ of $99.5 \%, S p$ of $99.7 \%$, and $A c c$ of $99.7 \%$, followed by a poly kernel with an average Se of $93.6 \%, \mathrm{Sp}$ of $99.6 \%$, and $\mathrm{Acc}$ of $98.9 \%$ from $\mathrm{CH}_{2}$.

Regarding the decision tree classifier, there are two parameters that can influence the performance: the maximum number of split (MNS) and the minimum leaf size (MLS). As for MNS, we optimized the DT classifier in the MATLAB environment and a MNS of 6 was considered as the optimal value. Thus, we investigated different numbers of leaves, i.e., 10, 20, 30, 40, and 50. Table 3 displays the classification results using DT. As can be observed, the best result was obtained by 20 leaves from $\mathrm{CH}_{1}$, with a mean $\mathrm{Se}$ of $100 \%, \mathrm{Sp}$ of $98.4 \%$, and $A c c$ of $98.7 \%$, followed by 30 leaves, with an average $S e$ of $100 \%$, Sp of $97.7 \%$, and Acc 
of $98.2 \%$. Compared to other two classifiers, the results obtained by DT seem to be more robust to the different channels.
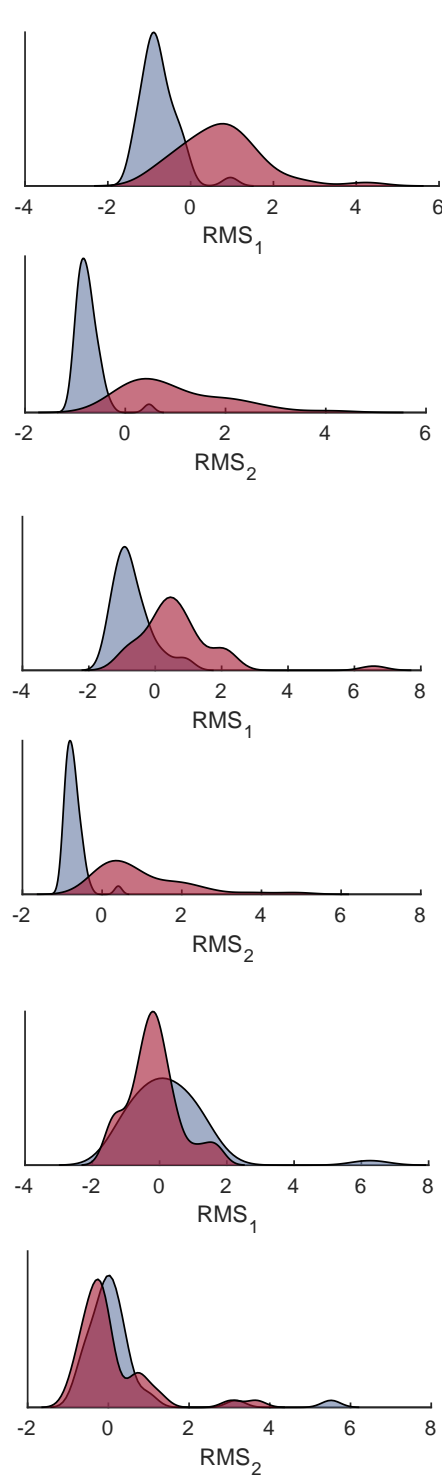
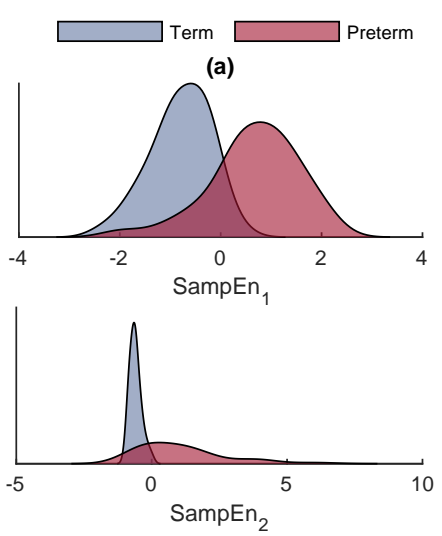

(b)
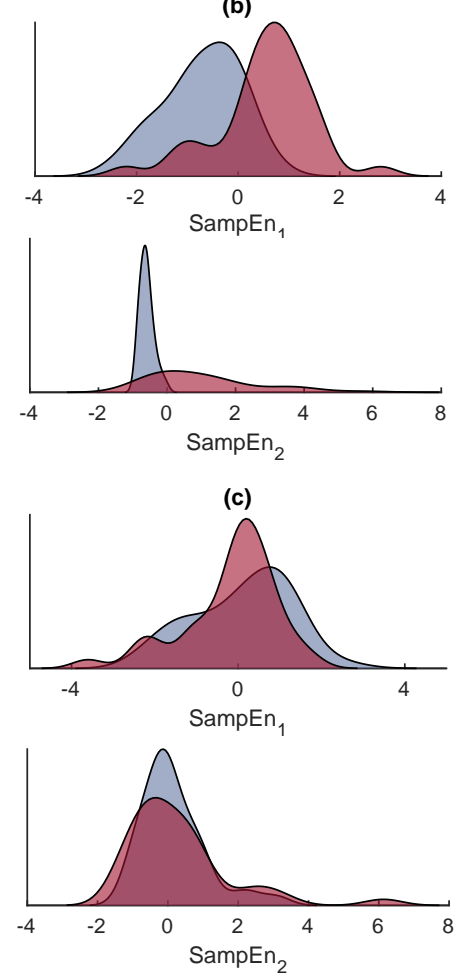
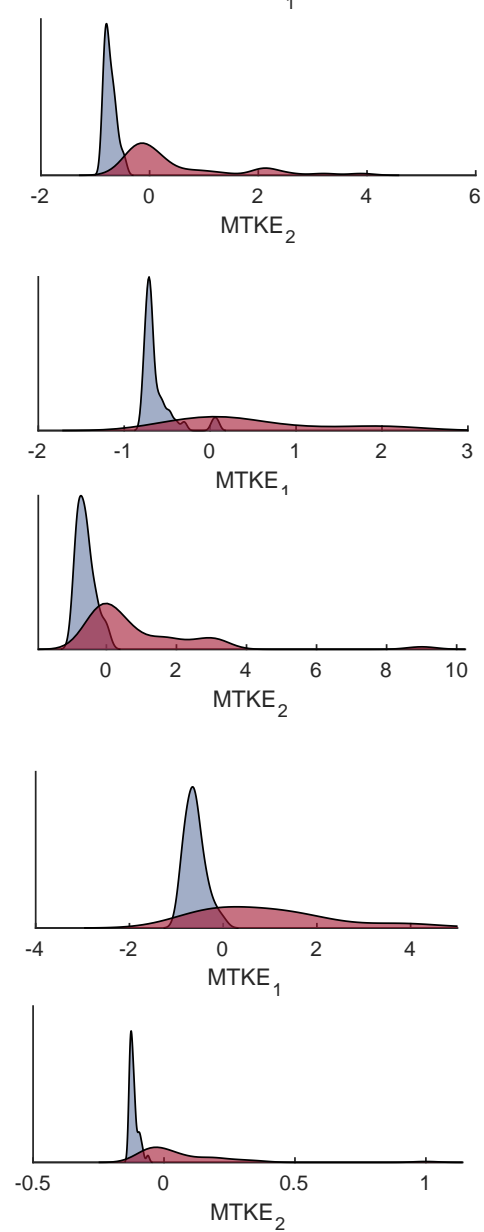

Figure 4. The distribution of the extracted features from IMF1 (first row) and IMF2 (second row) from (a) $\mathrm{CH}_{1}$, (b) $\mathrm{CH}_{2}$, and (c) $\mathrm{CH}_{3}$.

Table 2. SVM performance for different channel configurations. The best results are in bold.

\begin{tabular}{ccccc}
\hline Kernel & Channel & Se & Sp & Acc \\
\hline \multirow{3}{*}{ Linear } & CH1 & $77.5 \%$ & $98.4 \%$ & $95.8 \%$ \\
& CH2 & $78.9 \%$ & $98.5 \%$ & $96.0 \%$ \\
& CH3 & $85.0 \%$ & $99.6 \%$ & $97.7 \%$ \\
\hline \multirow{3}{*}{ RBF } & CH1 & $82.8 \%$ & $98.8 \%$ & $96.7 \%$ \\
& CH2 & $83.7 \%$ & $98.3 \%$ & $96.4 \%$ \\
\multirow{3}{*}{ Poly } & $\mathrm{CH} 3$ & $76.8 \%$ & $100 \%$ & $97.0 \%$ \\
& $\mathrm{CH} 1$ & $\mathbf{9 9 . 5} \%$ & $99.7 \%$ & $\mathbf{9 9 . 7} \%$ \\
& $\mathrm{CH} 2$ & $93.6 \%$ & $99.6 \%$ & $98.9 \%$ \\
& $\mathrm{CH} 3$ & $88.4 \%$ & $99.0 \%$ & $97.7 \%$ \\
\hline
\end{tabular}


Table 3. Decision tree performance for different channel configurations. The best results are in bold.

\begin{tabular}{ccccc}
\hline MLS & Channel & Se & Sp & Acc \\
\hline \multirow{2}{*}{10} & $\mathrm{CH} 1$ & $93.4 \%$ & $96.4 \%$ & $96.1 \%$ \\
& $\mathrm{CH} 2$ & $87.8 \%$ & $92.8 \%$ & $92.1 \%$ \\
& $\mathrm{CH} 3$ & $89.7 \%$ & $94.8 \%$ & $94.5 \%$ \\
\hline \multirow{2}{*}{20} & $\mathrm{CH} 1$ & $\mathbf{1 0 0} \%$ & $\mathbf{9 8 . 4} \%$ & $\mathbf{9 8 . 7} \%$ \\
& $\mathrm{CH} 2$ & $91.0 \%$ & $96.9 \%$ & $96.2 \%$ \\
& $\mathrm{CH} 3$ & $91.7 \%$ & $96.8 \%$ & $96.2 \%$ \\
\hline \multirow{3}{*}{30} & $\mathrm{CH} 1$ & $100 \%$ & $97.7 \%$ & $98.2 \%$ \\
& $\mathrm{CH} 2$ & $94.1 \%$ & $96.7 \%$ & $96.2 \%$ \\
& $\mathrm{CH} 3$ & $91 \%$ & $97.0 \%$ & $96.2 \%$ \\
\hline \multirow{2}{*}{40} & $\mathrm{CH} 1$ & $97.2 \%$ & $96.8 \%$ & $96.8 \%$ \\
& $\mathrm{CH} 2$ & $97.1 \%$ & $96.8 \%$ & $96.9 \%$ \\
& $\mathrm{CH} 3$ & $100 \%$ & $97.3 \%$ & $97.6 \%$ \\
\hline \multirow{2}{*}{50} & $\mathrm{CH} 1$ & $100 \%$ & $93.2 \%$ & $94.1 \%$ \\
& $\mathrm{CH} 2$ & $100 \%$ & $93.3 \%$ & $94.1 \%$ \\
& $\mathrm{CH} 3$ & $100 \%$ & $93.2 \%$ & $94.0 \%$ \\
\hline
\end{tabular}

According to Tables 1-3, the best performance results are obtained by SVM and DT classifiers using extracted features from $\mathrm{CH}_{1}$. Figure 5 compares the best obtained results in terms of $S e, S p$, and $A c c$ by all classifiers. As displayed, there is only a significant difference $(p<0.05)$ between $S e$ values of kNN vs. SVM and DT.

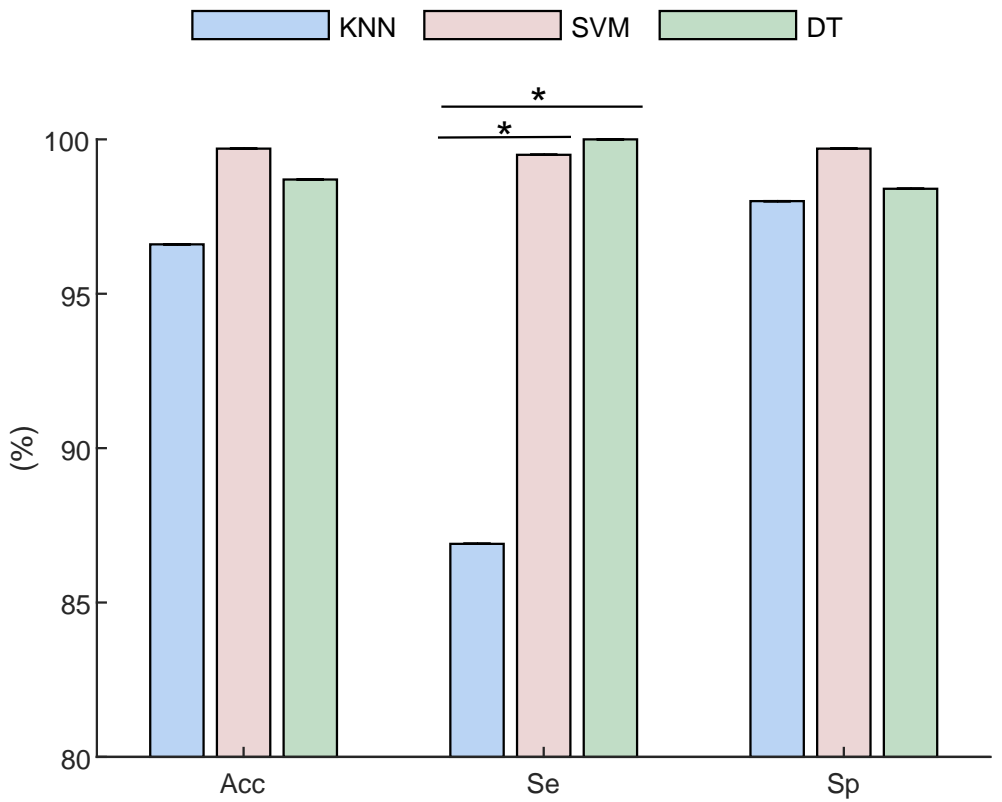

Figure 5. The performance comparison of all three classifiers. * stands for $p<0.05$.

The receiver operating characteristic (ROC) curves of all classifiers are shown in Figure 6. As can be observed, SVM achieved the highest AUC of 0.999, followed by DT and kNN, with 0.987 and 0.978 , respectively.

Table 4 compares our algorithm with state-of-the-art algorithms tested against the TPEHG DB database. The most noticeable advantage of our study is the use of original EHG records without synthetic data generation. In addition, our algorithm does not require feature ranking procedures. Instead, we employed three physiology-justified features which could properly discriminate term and preterm cases. Considering the similarity of feature extraction to our study, the best obtained results based on EMD analysis were 
achieved by the [39], with Se of $95.08 \%, S p$ of $97.32 \%$, and Acc of 96.25 . On the other hand, the resemblance of our results with [32] shows the applicability of the extracted features even when imbalanced EHG records were employed. Regardless the obtained results, due to low wearable complexity, i.e., single EHG channel, and computational cost, the proposed method could be integrated for using in home-based surveillance in indoor environments. Indeed, single-lead EHG monitoring paves the way for multimodal pregnancy monitoring in home-care scenarios, which is increasingly stressed in countries with the lowest population growth rates. The use of a single channel opens the possibility of selecting the best channel out of the three used in regular monitoring and of modifying the selection with changes of environmental conditions or the mother's position, which optimizes the monitoring quality-to-cost ratio and the accessibility of the method. Alternatively, a singlechannel EHG record may be considered as a part of a complex well-being record (including mother motion and fetus heart rate measurements) that documents the course of pregnancy while searching for possible threats.

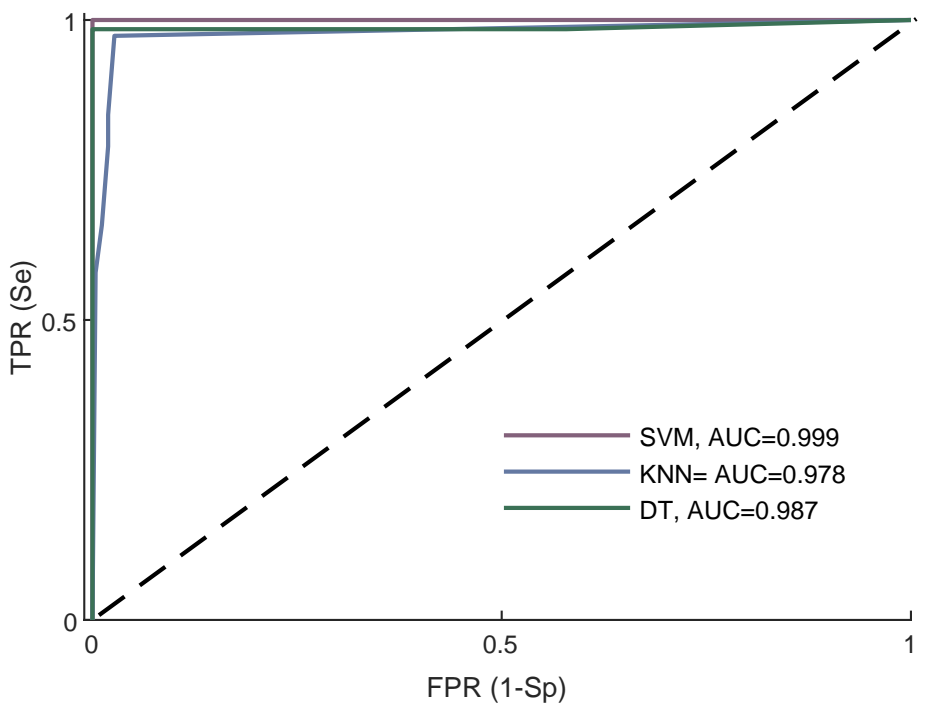

Figure 6. ROC of all classifiers with their best performance.

Table 4. The comparison of our study with sate-of-the-art algorithms. * means feature selection was performed before the classification.

\begin{tabular}{|c|c|c|c|c|c|c|c|c|}
\hline Work & Data Balancing & Channel & No. Data & Classifier & Acc & Se & Sp & AUC \\
\hline [27] & Yes (SMOTE) & $\mathrm{CH} 3$ & 262 term; 38 preterm & polynomial & - & $96 \%$ & $90 \%$ & 0.95 \\
\hline [28] & Yes (Min-Max) & $\mathrm{CH} 3$ & 150 term; 19 preterm & SONIA & $92.7 \%$ & $91.2 \%$ & $94.5 \%$ & 0.93 \\
\hline [29] & Yes (SMOTE) & $\mathrm{CH} 3$ & 262 term; 38 preterm & SVM & $87 \%$ & $96 \%$ & $79 \%$ & - \\
\hline [30] & Yes (SMOTE) & $\mathrm{CH} 3$ & 262 term; 38 preterm & Combined * & - & $91 \%$ & $84 \%$ & 0.94 \\
\hline [31] & Yes (ADASYN) & $\mathrm{CH} 1-3$ & 143 term; 19 preterm & $\mathrm{RF}^{*}$ & $93 \%$ & $89 \%$ & $97 \%$ & 0.962 \\
\hline [32] & Yes (ADASYN) & $\mathrm{CH} 1$ & 262 term; 38 preterm & SVM & $99.72 \%$ & $99.48 \%$ & $99.96 \%$ & - \\
\hline [37] & Yes (SMOTE) & $\mathrm{CH} 3$ & 262 term; 38 preterm & Adaboost & - & - & - & 0.986 \\
\hline [38] & No & $\mathrm{CH} 1-2$ & 26 term; 26 preterm & SVM * & $95.70 \%$ & $98.40 \%$ & $93 \%$ & 0.95 \\
\hline [39] & Yes (ADASYN) & $\mathrm{CH} 1-3$ & 262 term; 38 preterm & SVM * & $96.25 \%$ & $95.08 \%$ & $97.33 \%$ & - \\
\hline [33] & Yes (SMOTE) & $\mathrm{CH} 1-3$ & 275 term; 51 preterm & Ensemble * & $91.64 \%$ & $96.23 \%$ & $87.04 \%$ & 98.13 \\
\hline [5] & Yes (SMOTE) & $\mathrm{CH} 1-3$ & 275 term; 51 preterm & LDA * & $89.2 \%$ & $98.4 \%$ & $79.9 \%$ & 0.936 \\
\hline [34] & Yes (SMOTE) & $\mathrm{CH} 1-3$ & 262 term; 38 preterm & GBC & $85 \%$ & - & - & 0.91 \\
\hline [35] & Yes (Partition-Synthesis) & $\mathrm{CH} 1-3$ & 275 term; 51 preterm & SVM * & $91 \%$ & $89.0 \%$ & $93 \%$ & 0.97 \\
\hline$[40]$ & Yes (ADASYN) & $\mathrm{CH} 1-3$ & 262 term; 38 preterm & SVM * & $98.5 \%$ & $98.4 \%$ & $98.4 \%$ & - \\
\hline Ours & No & $\mathrm{CH} 1$ & 262 term; 38 preterm & SVM & $99.7 \%$ & $99.5 \%$ & $99.7 \%$ & 0.999 \\
\hline
\end{tabular}




\section{Conclusions and Future Works}

This paper presents an automatic algorithm for the accurate classification of term and preterm deliveries using a single EHG channel. The obtained results confirmed the adequacy of extracted features as no synthetic data-generation or feature-ranking algorithms were necessary. Indeed, our results suggested that employing features that properly characterize the contractions can avoid such extra processing. In future works, we should investigate (i) employing the categorical characteristics of each subject as the complementary features in addition to the proposed ones, (ii) the performance of employed features on other versions of filtered EHG signals with different cut-off frequencies (e.g., 0.3-1 Hz), (iii) the classification of EHG data based on the recorded weeks to investigate how far ahead preterm labor can be predicted, and (iv) the consistency of classification with the shortening of records.

Author Contributions: Conceptualization, S.M.F. and P.A.; methodology, S.M.F. and M.B.; validation, S.M.F. and M.S.; formal analysis, S.M.F.; investigation, S.M.F. and M.B.; resources, S.M.F. and M.S.; writing—original draft preparation, S.M.F.; writing—review and editing, P.A., M.B. and M.S.; visualization, M.S.; supervision, P.A.; funding acquisition, P.A. All authors have read and agreed to the published version of the manuscript.

Funding: This research was funded by the AGH University of Science and Technology in 2021 as research project No. 16.16.120.773.

Data Availability Statement: This database is publicly available at https:/ / physionet.org/content/ tpehgdb/1.0.1/, accessed on 20 October 2020.

Conflicts of Interest: The authors declare no conflict of interest.

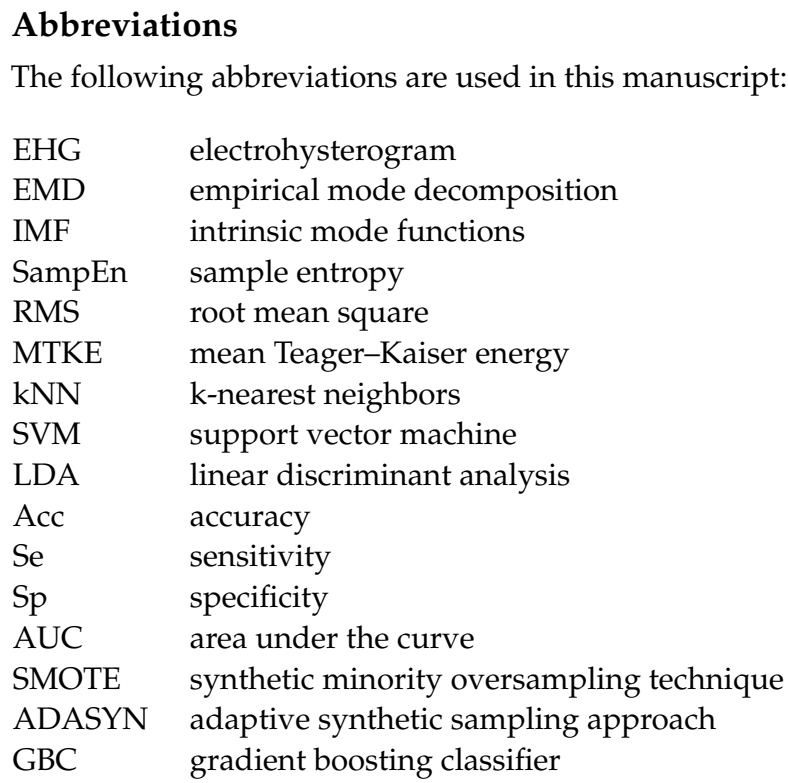

\section{References}

1. Frey, H.A.; Klebanoff, M.A. The epidemiology, etiology, and costs of preterm birth. Semin. Fetal Neonatal Med. 2016, $21,68-73$. [CrossRef]

2. Schleußner, E. The prevention, diagnosis and treatment of premature labor. Dtsch. Arztebl. Int. 2013, 110, 227-235. [CrossRef] [PubMed]

3. Iams, J.D.; Goldenberg, R.L.; Mercer, B.M.; Moawad, A.H.; Meis, P.J.; Das, A.F.; Caritis, S.N.; Miodovnik, M.; Menard, M.K.; Thurnau, G.R.; et al. The preterm prediction study: Can low-risk women destined for spontaneous preterm birth be identified? Am. J. Obstet. Gynecol. 2001, 184, 652-655. [CrossRef] [PubMed]

4. Available online: www.who.int (accessed on 15 September 2019). 
5. Nieto-del-Amor, F.; Beskhani, R.; Ye-Lin, Y.; Garcia-Casado, J.; Diaz-Martinez, A.; Monfort-Ortiz, R.; Diago-Almela, V.J.; Hao, D.; Prats-Boluda, G. Assessment of Dispersion and Bubble Entropy Measures for Enhancing Preterm Birth Prediction Based on Electrohysterographic Signals. Sensors 2021, 21, 6071. [CrossRef]

6. Horoba, K.; Wrobel, J.; Jezewski, J.; Kupka, T.; Roj, D.; Jezewski, M. Automated detection of uterine contractions in tocography signals-comparison of algorithms. Biocybern. Biomed. Eng. 2016, 36, 610-618. [CrossRef]

7. Barber, M.A.; Eguiluz, I.; Plasencia, W.; Medina, M.; Valle, L.; Garcia, J.A. Preterm delivery and ultrasound measurement of cervical length in Gran Canaria, Spain. Int. J. Gynecol. Obstet. 2010, 108, 58-60. [CrossRef]

8. Mourguesa, C.; Rossic, A.; Favrec, N.; Delabaereb, A.; Roszykd, L.; Sapind, V.; Debost-Legrandb, A.; Gallot, D. Fetal fibronectin test for threatened preterm delivery $48 \mathrm{~h}$ after admission: Cost-effectiveness study. Eur. J. Obstet. Gynecol. Reprod. Biol. 2019, 234, 75-78. [CrossRef] [PubMed]

9. $\quad$ Euliano, T.Y.; Darmanjian, M.T.N.M.T.S.; Mcgorray, S.P.; Euliano, N.; Onkala, A.; Gregg, A.R. Monitoring uterine activity during labor: A comparison of 3 methods. Am. J. Obstet. Gynecol. 2013, 208, 66.e1-66.e6. [CrossRef]

10. Hao, D.; Qiu, Q.; Zhou, X.; An, Y.; Peng, J.; Yang, L.; Zheng, D. Application of decision tree in determining the importance of surface electrohysterography signal characteristics for recognizing uterine contractions. Biocybern. Biomed. Eng. 2019, $39,806-813$. [CrossRef]

11. Jager, F.; Geršak, K.; Vouk, P.; Pirnar, Ž.; Trojner-Bregar, A.; Lucovnik, M.; Borovac, A. Assessing velocity and directionality of uterine electrical activity for preterm birth prediction using EHG surface records. Sensors 2020, 20, 7328. [CrossRef]

12. Mas-Cabo, J.; Prats-Boluda, G.; Ye-Lin, Y.; Alberola-Rubio, J.; Perales, A.; Garcia-Casado, J. Characterization of the effects of Atosiban on uterine electromyograms recorded in women with threatened preterm labor. Biomed. Signal Process. Control 2019, 52, 198-205. [CrossRef]

13. Mas-Cabo, J.; Ye-Lin, Y.; Garcia-Casado, J.; Alberola-Rubio, J.; Perales, A.; Prats-Boluda, G. Uterine contractile efficiency indexes for labor prediction: A bivariate approach from multichannel electrohysterographic records. Biomed. Signal Process. Control 2018, 46, 238-248. [CrossRef]

14. Xu, J.; Chen, Z.; Lou, H.; Shen, G.; Pumir, A. Review on EHG signal analysis and its application in preterm diagnosis. Biomed. Signal Process. Control 2022, 71, 103231. [CrossRef]

15. Batista, A.G.; Cebola, R.; Esgalhado, F.; Russo, S.; Reis, C.R.P.d.; Serrano, F.; Vassilenko, V.; Ortigueira, M. The contractiongram: A method for the visualization of uterine contraction evolution using the electrohysterogram, Biomed. Signal Process. Control 2021, 67, 102531. [CrossRef]

16. Babu, A.; Kumar, R. Optimized feature selection for the classification of uterine magnetomyography signals for the detection of term delivery. Biomed. Signal Process. Control 2020, 58, 101880.

17. Khalil, M.; Duchene, J. Uterine EMG analysis: A dynamic approach for change detection and classification. IEEE Trans. Biomed. Eng. 2000, 47, 748-756. [CrossRef]

18. Moslem, B.; Khalil, M.; Marque, C.; Diab, M.O. Complexity analysis of the uterine electromyography. In Proceedings of the 32nd Annual International Conference of the IEEE Engineering in Medicine and Biology Society (EMBC'10), Buenos Aires, Argentina, 31 August-4 September 2010; pp. 2802-2805.

19. Diab, A.; Hassan, M.; Marque, C.; Karlsson, B. Quantitative performance analysis of fourmethods of evaluating signal nonlinearity: Application to uterine EMG signals. In Proceedings of the Annual International Conference of the IEEE Engineering in Medicine \& Biology Society (EMBC'12), Doha, Qatar, 12-15 November 2012; pp. 1045-1048.

20. Fele-Zorz, G.; Kavsek, G.; Novak-Antolic, Z.; Jager, F. A comparison of various linear and non-linear signal processing techniques to separate uterine EMG records of term and preterm delivery groups. Med. Biol. Eng. Comput. 2008, 46, 911-922. [CrossRef]

21. Alamedine, D.; Khalil, M.; Marque, C. Parameters extraction and monitoring in uterine EMG signals. Detection of preterm deliveries. IRBM 2013, 34, 322-325. [CrossRef]

22. Ahmed, M.U.; Chanwimalueang, T.; Thayyil, S.; Mandic, D.P. A Multivariate Multiscale Fuzzy Entropy Algorithm with Application to Uterine EMG Complexity Analysis. Entropy 2016, 19, 2. [CrossRef]

23. Horoba, K.; Jezewski, J.; Matonia, A.; Wrobel, J.; Czabanski, R.; Jezewski, M. Early predicting a risk of preterm labour by analysis of antepartum electrohysterograhic signals. Biocybern. Biomed. Eng. 2016, 36, 574-583. [CrossRef]

24. Hoque, N.; Singh, M.; Bhattacharyya, D.K. EFS-MI: An ensemble feature selection method for classification. Complex Intell. Syst. 2018, 4, 105-118. [CrossRef]

25. Chawla, N.V.; Bowyer, K.W.; Hall, L.O.; Kegelmeyer, W.P. SMOTE: Synthetic Minority Over-sampling Technique. J. Artif. Intell. Res. 2002, 16, 321-357. [CrossRef]

26. He, H.; Bai, Y.; Garcia, E.A.; Li, S. ADASYN: Adaptive synthetic sampling approach for imbalanced learning. In Proceedings of the 2008 IEEE International Joint Conference on Neural Networks, Hong Kong, China, 1-8 June, 2008; pp. $1322-1328$.

27. Fergus, P.; Cheung, P.; Hussain, A.; Al-Jumeily, D.; Dobbins, C.; Iram, S. Prediction of preterm deliveries from EHG signals using machine learning. PLoS ONE 2013, 8, e77154. [CrossRef]

28. Hussain, A.J.; Fergus, P.; Al-Askar, H.; Al-Jumeily, D.; Jager, F. Dynamic neural network architecture inspired by the immune algorithm to predict preterm deliveries in pregnant women. Neurocomputing 2015, 151, 963-974. [CrossRef]

29. Smrdel, A.; Jager, F. Separating sets of term and preterm uterine EMG records. Physiol. Meas. 2015, 36, 341-355. [CrossRef] [PubMed] 
30. Fergus, P.; Idowu, I.; Hussain, A.; Dobbins, C. Advanced artificial neural network classification for detecting preterm births using EHG records. Neurocomputing 2016, 188, 42-49. [CrossRef]

31. Peng, J.; Hao, D.; Yang, L.; Dua, M.; Song, X.; Jiang, H.; Zhang, Y.; Zheng, D. Evaluation of electrohysterogram measured from different gestational weeks for recognizing preterm delivery: A preliminary study using random Forest. Biocybern. Biomed. Eng. 2020, 40, 352-362. [CrossRef] [PubMed]

32. Degbedzui, D.K.; Eminyüksel, M. Accurate diagnosis of term-preterm births by spectral analysis of electrohysterography signals. Comput. Biol. Med. 2020, 119, 103677. [CrossRef]

33. Nieto-del-Amor, F.; Prats-Boluda, G.; Martinez-De-Juan, J.L.; Diaz-Martinez, A.; Monfort-Ortiz, R.; Diago-Almela, V.J.; Ye-Lin, Y. Optimized Feature Subset Selection Using Genetic Algorithm for Preterm Labor Prediction Based on Electrohysterography. Sensors 2021, 21, 3350. [CrossRef]

34. Xu, J.; Chen, Z.; Zhang, J.; Lu, Y.; Yang, X.; Pumir, A. Realistic preterm prediction based on optimized synthetic sampling of EHG signal. Comput. Biol. Med. 2021, 136, 104644. [CrossRef]

35. Zhang, J.X.M.W.J.; Huang, Z.C.W.; Shen, G.; Zhang, M. Network theory based EHG signal analysis and its application in preterm prediction. IEEE J. Biomed. Health Inform. 2022, (early access).

36. Huang, N.E.; Shen, Z.; Long, S.R.; Wu, M.C.; Shih, H.H.; Zheng, Q.; Yen, N.C.; Tung, C.C.; Liu, H.H. The empirical mode decomposition and the hilbert spectrum for nonlinear and nonstationary Time Series Analysis. Proc. R. Soc. Lond. A 1971, 454, 903-995. [CrossRef]

37. Ren, P.; Yao, S.; Li, J.; Valdes-Sosa, P.A.; Kendrick, K.M. Improved prediction of preterm delivery using empirical mode decomposition analysis of uterine electromyography signals. PLoS ONE 2015, 10, e0132116. [CrossRef]

38. Sadi-Ahmed, N.; Kacha, B.; Taleb, H.; Kedir-Talha, M. Relevant features selection for automatic prediction of preterm deliveries from pregnancy electroHysterograhic (EHG) records. J. Med. Syst. 2017, 41, 1-13. [CrossRef] [PubMed]

39. Acharya, U.R.; Sudarshan, V.K.; Rong, S.Q.; Tan, Z.; Min, L.C.; Koh, J.E.; Nayak, S.; Bhandary, S.V. Automated detection of premature delivery using empirical mode and wavelet packet decomposition techniques with uterine electromyogram signals. Comput. Biol. Med. 2017, 85, 33-42. [CrossRef] [PubMed]

40. Khan, M.U.; Sajid, Z.; Sohail, M.; Aziz, S.; Ibraheem, S.; Naavi, S.Z.H. Electrohysterogram based Term and Preterm Delivery Classification System. In Proceedings of the 2020 First International Conference of Smart Systems and Emerging Technologies (SMARTTECH), Riyadh, Saudi Arabia, 3-5 November 2020; pp. 83-88.

41. Bhavsara, R.; Heliana, N.; Suna, Y.; Daveya, N.; Steffertb, T.; Mayor, D. Efficient Methods for Calculating Sample Entropy in Time Series Data Analysis. Procedia Comput. Sci. 2018, 145, 97-104. [CrossRef]

42. Boudraa, A.O.; Salzenstein, F. Teager-Kaiser energy methods for signal and image analysis: A review. Digit. Signal Process. 2018, 78, 338-375. [CrossRef]

43. Parsian, M. Data Algorithms; O'Reilly Media, Inc.: Newton, MA, USA, 2015.

44. Steinwart, I.; Christmann, A. Support Vector Machines; Springer: New York, NY, USA, 2008.

45. Smith, C.; Koning, M. Decision Trees and Random Forests: A Visual Introduction For Beginners: A Simple Guide to Machine Learning with Decision; Blue Windmill Media: Sheffield, UK, 2017.

46. Jager, F.; Libenšek, S.; Geršak, K. Characterization and automatic classification of preterm and term uterine records. PLoS ONE 2018, 13, e0202125. [CrossRef]

47. Shahrdad, M.; Amirani, M.C. Detection of preterm labor by partitioning and clustering the EHG signal. Biomed. Signal Process. Control 2018, 45, 109-116. [CrossRef] 